\title{
An Atypical Case of Progressive Spinal Amyotrophy (Type I) Likely Explained by Genetic Modifiers Case Report
}

\begin{abstract}
Marco Orsini'*, Marcos RG de Freitas², Acary Bulle Oliveira ${ }^{3}$, Carlos Henrique Melo Reis ${ }^{4}$, Antônio Marcos da Silva Catharino 4 , Jacqueline Fernandes do Nascimento ${ }^{4}$, Adriana Leico Oda ${ }^{3}$, Renata Castro ${ }^{4}$, Valéria Camargo Silveira ${ }^{4}$, Carlos Eduardo Cardoso ${ }^{5}$ and Mauricio Santanna Júnior ${ }^{6}$
\end{abstract}

${ }^{1}$ Iguaçu University - UNIG and Master's Program in Neurology - Vassouras University, Brazil

${ }^{2}$ Federal University of Rio de Janeiro, Brazil

${ }^{3}$ Federal University of São Paulo - Department of Neurology, Brazil

${ }^{4}$ Iguaçu University - UNIG, Brazil

${ }^{5}$ Vassouras University - Vassouras/RJ, Brazil

${ }^{6}$ Rio de Janeiro Federal Institute - IFRJ, Brazil

*Corresponding author: Marco Orsini, Iguaçu University - UNIG and Master's Program in Neurology - Vassouras University, Brazil.
Received Date: June 19, 2021

Published Date: July 09, 2021

\begin{abstract}
Introduction: Spinal muscular atrophy (SMA) is a group of inherited disorders associated with lower motor neuron depletion. Electromyography and muscle biopsy features of denervation were once the basis for diagnosis, but molecular testing for homozygous deletion or mutation of the SMN1 gene allows for an efficient and specific diagnosis. Although SMN1 has been identified as the SMA disease-determining gene, modifier genes mapped to 5q13 were affirmed to play a crucial role in determination of the disease severity and used as a target for SMA therapy. SMN2 copy number and other genetic modifiers in SMA patients can result in aprognosis outside the spectrum of the expected clinical evolutionin patients with homozygous deletion of SMN1.
\end{abstract}

Case Report: We report the case of a 64-year old patient who, since birth, had been diagnosed with type 1 SMA. However, the patient presented with a slower than expected disease progression for a type 1 SMA patient. Currently, although tetraparetic and functionally limited, the patient is still alive.

Results and Discussion: PCR analysis identified deletion in exons 7 and 8 of the SMN1 gene and confirmed the diagnosis of SMA.

Final Considerations: Our case report provides an overview of the clinical manifestations of SMA, comparing the findings of the neurological examination with the genetic panel. Understanding how genetic modifiers can affect the SMA disease phenol type will contribute to the basic understanding of the disease mechanism and further the development of new treatment strategies; guiding neurologists in diagnosis, prognosis, and treatment.

Keywords: SMA; Genetic; Prognosis; SMN; Motor neuron

\section{Introduction}

Spinal muscular atrophy (SMA) is a group of genetic disorders characterized by the degeneration of the anterior horn cells in the spinal cord and resultant symmetric proximal muscle atrophy and weakness. SMA is classified into five clinical grades based on age of onset and severity of the disease. Over the past several years, discoveries in the genetic panel have accelerated the 
identification of causative genes and provided essential advances in understanding the molecular and biological basis of SMA and insights into the selective vulnerability of lower motor neurons $[1,2]$. The most common SMA, accounting for over 95\% of cases, is an autosomal recessive disorder that results from a homozygous deletion or mutation in the $5 q 13$ survival of motor neuron (SMN1) gene.In combination with loss of SMN1, patients retain variable numbers of copies of a second similar gene, SMN2, which produces reduced levels of the survival motor neuron (SMN) protein that are insufficient for normal motor neuron function. There is an inverse relationship between SMN2 copy number and disease severity such that SMN2 is an accepted modifier gene of SMA. in addition toSMN2, several other genes are considered SMA disease phenotype modifiers, including SERF1, NAIP, GTF2H2, and TLL23.

\section{Case Report}

A64-year old retired bank officer male patient reported weakness, hypotonia, and diminished movement. The patient reported being restricted to a wheelchair, with the inability to independently sit or crawl since birth. Over the years, he became more dependent on others to perform basic and fundamental activities of daily living. Physical examination showed severe kyphoscoliosis, amyotrophy in all four limbs, bell-shaped chest, and drooping head syndrome (paresis of the neck extensors). On neurological examination: tetraparesis, abolished deep reflexes, amyotrophy, and joint shortening. The patient denied speech and swallowing problems. Additionally, the patient presented with severe respiratory dysfunction, corroborated by the values in the ventilatory function test (forced vital capacity). It is noteworthy the difficulties in lung expansion with "incarceration" of the left lung by scoliosis. Electroneuromyography showed damage to lower motor neurons.Motor conduction study demonstrated motor axons loss and excluded nerve conduction abnormalities of demyelinating motor neuropathy. Analyses performed using PCR techniques in the patient in the present study identified a deletion in exons 7 and 8 of the SMN1 gene and confirmed the diagnosis of SMA.

\section{Discussion}

Although PCR testing demonstrated deletions in exons 7 and 8 of the SMN1 gene, the patient in question did not undergo the MLPA (Multiplex Ligation-Dependent Probe Amplification). The number of SMN2 genes may vary, and a higher SMN2 copy number is associated with less-severe symptoms of SMAvia the dosage compensation effect. Ideally, the MLPA test should be performed in order to quantify the number of SMN2copies. Higher SMN2 copy number is our main hypothesis for the dissociation between the natural history of this patient's disease and the genetic result. A commonly used polymerase chain reaction/restriction fragment length polymorphism (PCR-RFLP) assay can be used to detect only a homozygous absence of SMN1 exon 7. SMN gene dosage analyses, which can determine the copy numbers of SMN1 and SMN2, have been developed which in addition to detecting homozygous absence of SMN1, both estimates the number of SMN2 and identifies individuals with heterozygous absence of SMN1 who, may potentially have SMA due to an intragenic mutation in the remaining SMN1 copy $[3,4]$.

Multiplex ligation-dependent probe amplification (MLPA) is a modern quantitative molecular method. Applied in SMA cases, it improves diagnostics by simultaneously identifying the number of copies of several target sequences in the SMN1 gene and in nearby genes4. Unfortunately, our patient did not have this method when he underwent genetic testing,which probably made him retain his molecular diagnosis of type 1 SMA, incompatible with his clinical condition. MLPA provides a simple, rapid, and accurate method of simultaneously detecting homozygous deletions and heterozygous deletions in a single assay for both SMN1 and SMN2 genes [5]. Another possibility is a Clarity $^{\mathrm{TM}}$ digital PCR (dPCR) system. Genomic DNA samples collected from whole blood, amniotic fluid or dried blood stains can be analyzed using the Clarity ${ }^{\mathrm{TM}}$ Digital PCR (dPCR) system to determine the copy numbers of the SMN1 and SMN2 genes. Two hundred and fourteen clinical specimens determined by the qPCR-based method were enrolled and used to establish cut-off ranges for individual categories of SMA carriers and unaffected SMA patients. After defining the cut-off range for each group, 12 samples were analyzed by method based on dPCR and MLPA (multiplex binding dependent probe amplification), the current gold standard test for SMA and 100\% (total) concordant results between the two test methods were performed. The CSB SMA detection kit combined with the dPCR platform provides a robust and accurate approach to distinguishing unaffected individuals, SMA carriers and SMA patients. This rapid molecular diagnostic method can be adapted for pre-pregnancy eugenic inspection, prenatal testing, and newborn screening and help from physicians or genetic counselors to improve the incidence of SMA in the population [6].

\section{Final Considerations}

Spinal muscular atrophy (SMA) is a common autosomal recessive disorder which has been considered as the second most common genetic cause of infant death, with an estimated prevalence of 1 in 10,000 live births. Comprehensive SMA genetic testing, comprising PCR-RFLP assay, SMN gene dosage analysis, and linkage analysis, combined with appropriate genetic risk assessment and genetic counseling, offers the most complete evaluation of SMA patients and their families at this time.

\section{Acknowledgement}

None.

\section{Conflict of Interest}

No conflict of interest.

\section{References}

1. Ji X, Liu XQ Shen JW, Li XH, Tao J (2010) Genetic tests and clinical reevaluation of 85 children with suspected spinal muscular atrophy. Zhonghua Er Ke Za Zhi 48(6):425-30. 
2. Farrar MA, Kiernan MC (2015) The Genetics of Spinal Muscular Atrophy: Progress and Challenges. Neurotherapeutics 12(2): 290-302.

3. Jiang J, Huang J, Gu J, Xiaoshu Cai 4, Hongyu Zhao, et al. (2019) Genomic analysis of a spinal muscular atrophy (SMA) discordant family identifies a novel mutation in TLL2, an activator of growth differentiation factor 8 (myostatin): a case report. BMC Med Genet 20: 204.

4. Arkblad EL, Darin N, Berg K, Kimber E, Brandberg G, et al. (2006) Multiplex ligation-dependent probe amplification improves diagnostics in spinal muscular atrophy. Neuromuscul Disord 16(12): 830-838.
5. Tomaszewicz K, Kang P, Wu BL (2005) Detection of homozygous and heterozygous SMN deletions of spinal muscular atrophy in a single assay with multiplex ligation-dependent probe amplification. Beijing Da Xue Xue Bao Yi Xue Ban 37(1): 55-7.

6. Wang KC, Fang CY, Chang CC, Chiang CK, Chen YW (2021) A rapid molecular diagnostic method for spinal muscular atrophy. J Neurogenet 35(1): 29-32. 